www.jmscr.igmpublication.org

Impact Factor 5.244

Index Copernicus Value: 5.88

ISSN (e)-2347-176x ISSN (p) 2455-0450

crossref DOI: http://dx.doi.org/10.18535/jmscr/v4i4.10

\title{
Non-Specific Inflammatory Intra-Thoracic Mass Mimicking Malignancy: A Case Report
}

\author{
Author \\ Satya Prakash Meena', Biplab Mishra ${ }^{2}$, Rajinder Parshad ${ }^{3}$, Mohit Joshi ${ }^{4}$, \\ Sudheer Arava ${ }^{5}$, Sivasai Krishanaparsad K ${ }^{6}$, Mufaddal Kazi ${ }^{7}$ \\ ${ }^{1}$ Senior Resident, Department of Surgery, AIIMS, Delhi, India \\ Email: drsatyaprakash00@gmail.com \\ ${ }^{2}$ Add. Prof, Department of Surgery, AIIMS, Delhi, India \\ Email: biplabaiims@gmail.com \\ ${ }^{3}$ Prof. Department of Surgery, AIIMS, Delhi, India \\ Email: rajinder.parshad@gmail.com \\ ${ }^{4}$ Asst.Prof, Department of Surgery, AIIMS, Delhi, India \\ Email:drmohitjoshi@gmail.com \\ ${ }^{5}$ Asst. Prof, Department of Pathology, AIIMS, Delhi, India \\ Email:aravaaiims@gmail.com \\ ${ }^{6}$ Senior Resident, Department of Surgery, AIIMS, Delhi, India \\ Email: kskrishnaprasad@yahoo.com \\ ${ }^{7}$ Senior Resident, Department of Surgery, AIIMS, Delhi, India \\ Email: mufaddalkazi@gmail.com
}

\begin{abstract}
A 42-year-old man presented with long standing history of cough with hemoptysis. The clinical and radiological features were suspicious for a low grade malignancy. An enbloc resection of tumor with adherent chest wall and diaphragm was performed. The histological finding of chronic non-specific inflammation surprised us. Although few benign intra thoracic mass lesions are known to mimic lung cancer but a non- specific inflammatory mass lesion in thorax masquerading as malignancy has never been reported. We report our experience with this unique clinical entity and also discuss the diagnostic and therapeutic challenge we faced in managing this patient.

Keywords- Cough, Hemoptysis, Nonspecific inflammatory mass, Thorax, Malignancy
\end{abstract}

\section{Introduction}

Chronic Inflammatory mass mimicking malignancy is rare. There are reports in literature where Inflammatory myofibroblastic tumor, inflammatory pseudotumor or chronic fungal abscesses have posed diagnostic dilemma being indistinguishable from lung malignancy clinically and radiologically but reports of chronic inflammatory mass mimicking malignancy is scarce ${ }^{1,2,11,12}$. It often occur as a result of repeated thoracic interventions for infective cause, most likely after empyema or lung abscess. These Interventions are usually repeated thoracocentesis, ICD insertion, attempts at biopsy and even 
thoracotomies. As a result of repeated interventions, excessive inflammatory response occurs, that result in chronic fibrosis which may mimic as an intrathoracic malignant mass.

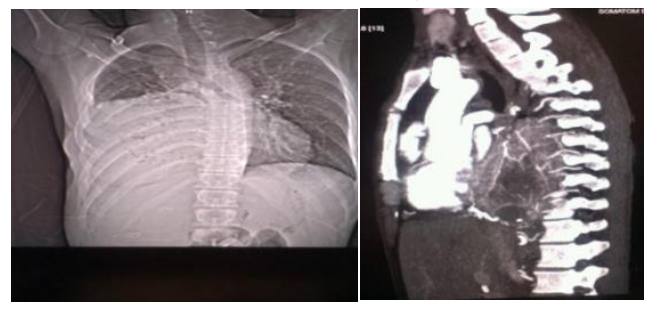

(Fig 1)

(fig.2)

\section{Case Report}

A gentleman aged 42 years, had cough and haemoptysis since 9 years, and mild continuous dull aching pain in right side of chest for 6 years. For these complaints, he was evaluated and a diagnosis of right empyema thoracis was made. $\mathrm{He}$ underwent repeated thoracocentesis and intercostal chest tube (ICD) insertion over variable period of time. He also underwent video assisted thoracoscopic surgery (VATS) decortication and open thoracotomy with decortications for chronic empyema. These interventions did not improve his symptoms and over the period he lost $15 \mathrm{kgs}$ of weight. He then presented to us in Surgical emergency with massive hemoptysis. On admission, the patient was pale and had pulse rate of 110/ min, BP 80/50mmHg and RR 24/min. He was afebrile and had pedal edema. The rest of the general physical examination was unremarkable. He had $40 \mathrm{kgs}$ weight with BMI of 14.3 . There was no history of fever, trauma, syncope, tuberculosis, hypertension, diabetes mellitus and family history of malignancy. On chest examination, patient had multiple scars, pus discharging sinuses, crowding of ribs, mild tenderness, and dull note over right middle and lower chest. The breath sounds were decreased in right lower chest. On routine investigations, his haemoglobin was $4.4 \mathrm{gm} \%$ and albumin was $2.7 \mathrm{gm} \%$. The rest of the haematological and biochemical parameters were within normal limits. There was no suggestion of bleeding disorder. The chest X-Ray showed a well defined homogenous mass lesion involving mid and lower zone of right hemithorax abutting the chest wall, silhouetting the right heart border. Cardiophrenic angle was obscured (Fig 1).A CECT scan Chest was suggestive of a large heterogeneous mass of size $13 \times 10 \times 13 \mathrm{~cm}$ with air foci in right middle and lower lobe and crowding of ribs, however the underlying ribs were normal. There were few foci of calcification along the right pleura suggestive of the chronicity of the lesion (Fig 2). Large number of dilated tortuous blood vessels were seen supplying the mass. The study was supplemented with angiography that revealed multiple tortuous, hypertrophied bronchial and intercostal vessels, collateral from right inferior phrenic artery, and internal mammary artery abnormally supplying the mass (Fig 2,3,4,). In view of significant haemoptysis, few of these vessels were embolised.

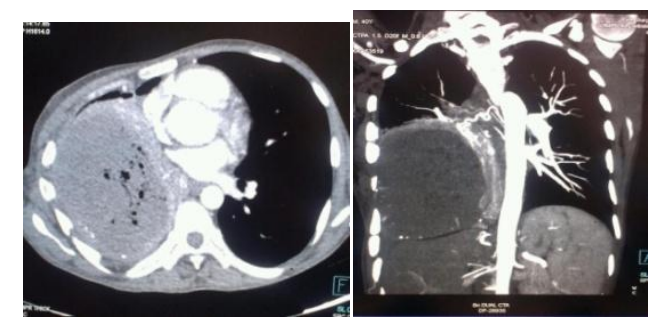

(Fig 3)

(Fig 4)

A USG guided biopsy from the lesion showed inflammatory infiltrates. Sputum culture for AFB was negative and discharge from chest wall sinuses grew acenatobactor. A Bronchoscopy was performed, that showed edematous right upper lobe bronchus. Bronchus intermedius was found compressed extrinsically with edematous mucosa, that was bleeding on touch. On the basis of these clinical, radiological and histological findings, a diagnosis of a vascular thoracic tumor likely benign was made, although the possibility of a low grade malignant neoplasm was not ruled out. Patient was operated on semi-emergent basis in view of significant haemoptysis that did not responded to embolisation. Anteroterolateral thoracotomy was performed that revealed a large highly vascular firm tumor closely abutting the heart and large vessels. This mass was fixed to overlying chest wall and a part of diaphragm and looked like a locally invasive neoplasm. Right 
pneumonectomy with enbloc excision of mass with adherent chest wall (rightt 4,5,6 ribs, part of sternum) and diaphragm was done (Fig 6). The chest wall was then reconstructed using myocutaneous flap.

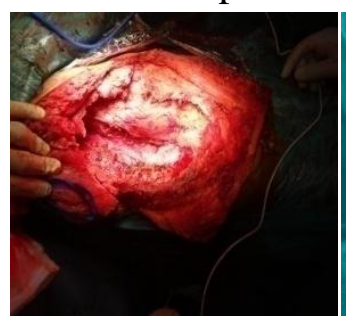

(Fig 5)

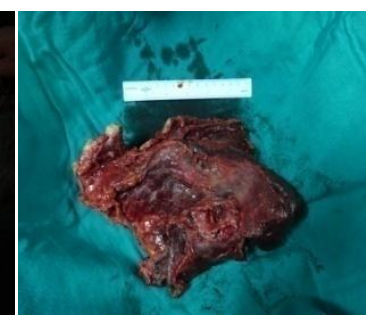

(Fig 6)
Patient received post operative care in ICU for 7 days. He was extubated on day 5 . The chest tube was removed on day 10 . He made a complete although slow recovery. Patient was discharged 20 days after surgery. At two month of follow up, he is asymptomatic. The biopsy of specimen suggested a well-defined, firm, lobulated parenchymal mass with central necrosis. There were dense mixed inflammatory cells, inflammation in the alveolar space and interstitium. There also was significant interstitial lymphoid follicle formation and intervening blood vessels showed medial hypertrophy with perivascular fibrosis. Adjacent pleura show dense fibrosis and hyalinization. There was no evidence of dysplasia, malignancy, granulomas or fungal profiles . These changes were suggestive of non-specific chronic inflammation.

\section{Discussion}

Chronic inflammatory thoracic mass mimicking lung cancer is rare. Benign inflammatory mass lesions like IMFT, pseudotumor and chronic inflammation in underlying pulmonary fungal lesions are known to present with clinical and radiological features that are indistinguishable from lung carcinoma, however to the best of our knowledge chronic non-specific inflammation masquerading as lung cancer has not been reported so far. In IMFT and pseudotumor, the lesion occur secondary to unregulated growth of inflammatory cells, the triggering of which is obscure $^{4}$. Largely the patients are asymptomatic and this lesion is picked up as an incidental finding on imaging done for some other reasons ${ }^{1,5}$. Although at times the patients may present with cough with or without hemoptysis that may raise the suspicion of malignancy. Similarly pulmonary fungal infections like coccidomycosis, blastomycosis, cryptococosis, histoplasmosis and aspergillosis have also been reported to mimic thoracic malignancy clinically and radiologically ${ }^{11,12}$. Our patient had persistent cough with hemoptysis and chest pain for long duration that made us entertain the possibility of a chronic benign lesion clinically. Repeated thoracic interventions in our patient may have incited inflammatory reaction that continued over years resulting in the formation of a mass like lesion. The persistent inflammation that only progressed with time may also have caused hypertrophy of the draining vessels and neovascularisation resulting in formation of large collateral vessels making the tumor so vascular. Making a preoperative diagnosis of non-specific inflammatory mass lesion was not possible although the chronicity of the lesion was suggestive of a benign process. Moreover on imaging studies a neoplastic process like a low grade malignant lesion could not be excluded confidently. The intraoperative findings of locally invasive mass lesion (to the chest wall and diaphragm) further consolidated our suspicion of a low grade malignancy. Assuming this possibility we resected the tumor enbloc with the adherent structures that was a surgical challenge in a poorly optimized patient who was not a good surgical candidate. It was only on histopahology that the true benign nonspecific inflammatory nature of the lesion was revealed. The histological findings were not suggestive of IMFT or pseudotumor. There were no mycotic elements that ruled out the possibility of a fungal lesion. We highlighted this case as it is rare and adds on to the known benign causes that may mimic lung carcinoma. Treating physicians and surgeons should be aware of the existence of this entity so as to entertain it as a differential diagnosis and appropriate management. 


\section{JMSCR Vol||04||Issue||04||Page 10022-10025||April}

\section{Conclusion}

Chronic non-specific inflammatory lesion in chest may mimic lung cancer. Awareness of this entity is important for considering a pre operative diagnosis and appropriate management.

\section{References}

1. Pattinato $\mathrm{G}$,Manival ,J.C.,Derosa $\mathrm{N}$ and Dehner ,L.P. Imflammatory myofibroblastic tumor -clinico pathological study of cases with immunohistochemical and ultrastructural observations. Am J Clin 1990,94,538-546

2. Melloni $\mathrm{G}$,Caretta, $\mathrm{A}$,Ciriaco ,P ,Arrigoni, $\mathrm{G}$,Fieschi S Rizzo $\mathrm{N}$ et al. Imflammatory pseudotumor of the lung in adult .Ann Thorac Surg 2005, 79.,426-432

3. Sakural H , Hasogawa ,t,Watanabe , S.Suzuki k Imflammatory myofibroblastic tumor of the lung Europian J CardioThoracic surg 2004,25, 155-159.

4. Shininchi Takeda, Yasuyo onishi ,Tomohiro etal:Clinical spectrum of pulmonary imflammatory myofibroblastic tumor. In cardiovasc Thorac Surg (2008) 7 (4)629-633

5. Agrons ,G A ,Rosado de Christenson ,M.L.etal Pulmonary imflammatory pseudotumor radiological features ,Radiology 1996 ,208,511-518

6. Taichiro $G$,KumiA ,Arafurmi $M$ and Ryoichi K Surgery for recurrent imflammatory pseudotumor of the lung .Word J Surg Oncol $2011,9,133$

7. Cerfolio R J Allen ,M.S. Nascimento A.G. et al Imflammatory pseudotumor of the lung :Thoracic Surg :1999,67,933-936

8. (8)Matsubara, O,Tan liu.N.S.Kenney, R.M. And Mark: A: Imflammatory pseudotumor of the lung progression from organizing pneumonia to fibrous histocytoma or to plasma cell granuloma in a 32 cases. Mum patoi $1988,19,807-814$

9. Siosman D.O ,Spilopoulos ,A, Lamoine , R.,Besse etal :Quantitative metabolic
P.E.T.Imaging of a plasma cell granuloma , J Thoracic Imaging 1994, 9, 116-119

10. Kobashi Y ,Fukuda ,M ,Nakata M ,Oka M Etal :Imflammatory pseudotumor of lung Clinico pathological analysis inseven adult patients :Int J Oncol 2006, 11, 461-466

11. Rolston KV, Rodriguez S,Dholakia N: Pulmonary infection mimicking cancer : a retrospective ,three year review(1997) study of texas, Support care cancer 5(2) 90-93.

12. Schweigert M, Dubecz A, Beron M etal : Pulmonary infection initiating lung cancer :clinical presentation and therapeutical approach.Ir J Med Sci 182(1) :73-80 . 